\title{
Guest Editorial \\ Evolving Fuzzy Systems-Preface to the Special Section
}

\section{Evolving FuZZY Systems AS AN EMERGING TOPIC IN FUZZY SYSTEM THEORY AND APPLICATIONS}

I $T$ IS a well-recognized fact that the theory of fuzzy sets and systems, for the last four decades after the seminal paper by Professor Zadeh [1], has demonstrated its remarkable ability to go beyond conventional information representation. It resulted in a wide range of new formulations of practical problems, such as fuzzy control, fuzzy clustering and classification, fuzzy modeling, and fuzzy optimization [2]. Historically, the design of the fuzzy systems has been initially assumed to be centered on expert knowledge [3]. During the 1990s, a new trend emerged [4], [5] that offered techniques to make use of the experimental data. This data-centered approach can be used to enhance and validate the existing expert knowledge or can also be used to substitute its lack (as is the case with autonomous systems, for example). Neurofuzzy and hybrid learning systems were introduced, where fuzzy representation was integrated into a neural learning architecture to bring linguistic meaning of the learned information [5].

An important research challenge today is to develop such techniques and methodologies that allow highly adaptive systems to be self-developed from streaming data (collected in real-time from sensory inputs, the Internet, the environment, or the system monitoring itself). Conventional adaptive systems known from control theory are usually applicable to a very narrow range of conditions (usually linear systems, specific Gaussian assumptions for noise and signals, etc.). Moreover, they address the problems of adjustment of the system parameters and coefficients only and normally consider the system structure as a given prefixed entity. Fuzzy (and neurofuzzy) systems offer an enormous area for exploitation with the concept of fuzzily interlinked multimodel substructures that can be simple (e.g., linear) and thus, tractable. The newly emerging concept of dynamically evolving structures, which was, to some extent, already applied to neural networks [6], brought a powerful new concept of evolving fuzzy systems (EFSs). EFSs combine: 1) the interpolation abilities of the fuzzy systems; 2) their flexibility; 3 ) the linguistic interpretability of the fuzzy systems; and 4) the adaptive feature of the online learning techniques.

This new topic was introduced during the last decade [7], [8], [10] and quickly numerous applications to problems of modeling, control, prediction, classification, and data processing in a dynamically changing and evolving environment were also developed, including some successful industrial applications [9]-[17].

Digital Object Identifier 10.1109/TFUZZ.2008.2006743
One can formulate EFS as a synergy between fuzzy (or neurofuzzy) systems as expandable (evolvable) structures for information representation and real-time methods for machine learning. EFS are addressing nonstationary processes by computationally efficient algorithms for real-time applications. They consider a dynamically evolving family of locally valid subsystems that can represent different situations or operating conditions. As Alan Turing said, "the Global order emerges from the chaos of local interactions." EFS aim at lifelong learning and adaptation, and self-organization (including system structure evolution) in order to adapt to (unknown and unpredictable) environments. EFS also address the problems of detecting temporal shifts and drifts in the data patterns, adapting the learning scheme itself in addition to the evolution of the structure and parameters of the system.

There is a growing demand for technical implementations enabled with such capabilities that an EFS offers in many application domains, such as: 1) autonomous vehicles and systems [19]; 2) intelligent sensors and agents [14]; 3) finance, economics, and social sciences [17]; 4) transportation systems [15]; 5) advanced manufacturing and process industries such as chemical, petrochemical, etc. [20]; and 6) biomedicine, bioinformatics, and nanotechnology [18].

The research effort and the number of publications in the area of EFSs are constantly growing through a series of organized events such as dedicated symposia, workshops, and special sessions in leading international conferences related to different facets of computational intelligence, and those were the motivating factors to organize this special section.

\section{Papers Submitted And Selected for the Special SECTION-AN OVERVIEW}

As a result of the call, over 20 full-length papers were submitted, which is a significant pool of contributions in an area that is in its infancy. A few of the papers were not directly relevant to the call, which was deliberately formulated quite narrowly (aiming to select the relatively small number of new contributions that are focused and strong, rather than to give a broad picture and have fuzzy boundaries of the area that are yet to be more widely recognized). A few papers were caught by the ambiguity of the adjective "evolving" in the title in the sense as established in the computational intelligence area, where the perception of this term is related to evolutionary paradigms such as mutation, crossover, and selection, while the context in which the term is used in this special section and in this emerging area is the one that is related to the dynamic development of a system. 
Out of the papers that cover different aspects of online design of EFSs, seven papers were finally selected, which passed all rounds of anonymous review process typical for the TRANSACTIONS. This number includes one paper coauthored by one of the Guest Editors and following the TRANSACTIONS' review protocol, this paper was handled by an Associate Editor, picked up by the Editor-in-Chief directly. Thus, we followed strictly the review procedure of the IEEE TRANSACTIONS ON FUZZY SYSTEMS.

The first paper in this special issue, titled "FLEXFIS: A Robust Incremental Learning Approach for Evolving TakagiSugeno Fuzzy Models," by Edwin Lughofer, is a typical representative of the EFS area. It introduces a new approach to online design of a specific type of Takagi-Sugeno fuzzy models, the so-called fuzzy basis function networks proposed by Wang and Mendel. This is another example of the emerging plethora of data-centered approaches to tackle online incremental learning and self-adaptation in fuzzy systems. The specific of this approach is that it stems from the well-known vector quantization (VQ) clustering for developing the premise part of the fuzzy systems. An evolving version of VQ (eVQ) is used in combination with the fuzzily weighted recursive least-squares method.

In the paper, "A Self-Evolving Interval Type-2 Fuzzy Neural Network With Online Structure and Parameter Learning," by Chia-Feng Juang and Yu-Wei Tsao, a new self-evolving online structure, which can also be seen as a neurofuzzy system or eqivalently as a Takagi-Sugeno-type fuzzy system, is proposed. The main distinctive feature of this paper is that the system assumes interval type-2 fuzzy sets that supersede the well-known "conventional" or type-1 fuzzy sets. In a manner typical for the evolving systems, both the system structure and the system parameters are self-developed (evolved) from data; this process can start from "scratch" (i.e., from an empty rule base) and is driven by an online clustering method. An efficient fuzzy set reduction method is proposed and the learning is performed using a rule-ordered Kalman filter.

The paper, "Incremental Evolution of Fuzzy Grammar Fragments to Enhance Instance Matching and Text Mining" by Trevor Martin, Yun Shen, and Ben Azvine, differs from the other papers in its emphasis on text mining and on the use of a fuzzy grammar. The area of extracting structured data from sections of unstructured text is of great importance for mining data that are streamed from the Internet, multimedia communications, etc. The novel approach presented in this paper falls into the group of incremental learning methods, since the structure of the fuzzy grammar is not known beforehand, but rather emerges from the streams of data by online learning. Results that surpass an offline genetic programming technique, while working online, were achieved on data streams collected from telephone conversations, e-mails, uniform resource locators, and others.

The paper "An Evolving Fuzzy Predictor for Industrial Applications" by Wilson Wang and Josip Vrbanek, introduces a recursive version of the popular Levenberg-Marquardt search algorithm that is used to identify in an online mode the parameters of the evolving fuzzy system. Convergence of the proposed learning method has been proved. The system structure is evolved through online clustering. The proposed scheme is vali- dated on benchmark time series and on a problem of monitoring machinery conditions and material property. Results suggest that the proposed evolving fuzzy system is an effective and accurate forecasting tool that can capture the system's dynamic behavior and track the change in the system's characteristics.

The paper "Fully Evolvable Optimal Neurofuzzy Controller Using Adaptive Critic Designs” by Salman Mohagheghi, Ganesh K. Venayagamoorthy, and Ronald G. Harley, demonstrates an application of EFSs to control. The core of the controller consists of a zero-order Takagi-Sugeno fuzzy rule base. In addition to this, core heuristic dynamic programming can be applied that can ensure near-optimal control under various operating conditions. The whole control scheme has been demonstrated to perform efficiently and to lead to lower capital investment.

The paper "Evolving Fuzzy-Rule-Based Classifiers from Data Streams" by Plamen Angelov and Xiaowei Zhou, introduces a new approach to classification of streaming data by the use of EFSs. The proposed approach, called evolving Classifier (eClass), includes different architectures and online learning methods. This family includes eClass0, which is very similar to the conventional classifiers where the consequent part of the fuzzy sets indicates the class label. eClass 1 is a newly proposed method for regression over the features that has an option for a multiclass output. An important property of eClass is the ability to start classifying and learning "from scratch" and to learn from examples. Not only the rule number but also the number of classes does not need to be prespecified and fixed. This was demonstrated to be very efficient on the data from intrusion detection, an example given at the KDD99 competition where eClass was able to learn from several hundreds to few thousands of data samples compared to the whole dataset of over five million samples. The proposed approach addresses the practical problems of the classification of streaming data (video, speech, and sensory data generated from robotic, advanced industrial applications, financial and retail chain transactions, and intruder detection, etc.).

Finally, the paper "Efficient Self-Evolving Evolutionary Learning for Neuro-Fuzzy Inference Systems" by Cheng-Jian Lin, Cheng-Hung Chen, and Chin-Teng Lin represents the evolutionary fuzzy systems with an approach called Self-Evolving Evolutionary Learning Algorithm (SEELA), which combines particle-swarm optimization for both structure and parameter identification of (neurofuzzy) systems. Furthermore, a hybrid called cooperative particle-swarm optimization is introduced. This approach where the learning is offline has been applied to time series and nonlinear control. It demonstrates the difference in the principles of evolving and evolutionary as described before.

\section{ACKNOWLEDGMENT}

The Guest Editors would like to thank numerous authors who responded to the call and anonymous reviewers who, with their expert and visionary analyses, made it possible not only to select a few successful papers, but also to shape the special issue and to direct the future research on EFSs. The guest Editors would like to thank the Editor-in-Chief, Prof. Nikhil Pal, for his highly 
professional attitude and dedication to the quality of the journal and for the opportunity to shed light on this emerging topic of research.

\author{
Plamen ANGELOV, Guest Editor \\ Intelligent Systems Research Laboratory \\ Lancaster University \\ Lancaster LA1 4WA, U.K. \\ p.angelov@lancaster.ac.uk
}

\author{
DIMITAR FILEV, Guest Editor \\ Ford Motor Company \\ Ford Research \& Advanced Engineering \\ Dearborn, MI 48121 USA \\ dfilev@ford.com
}

NiKOla KaSABOV, Guest Editor

Knowledge Engineering and Discovery Research Institute (KEDRI), Auckland University of Technology,

Auckland 1142, New Zealand

nkasabov@aut.ac.nz

\section{REFERENCES}

[1] L. A. Zadeh, "Fuzzy sets," Inf. Control, vol. 8, pp. 338-353, 1965.

[2] R. R. Yager and D. P. Filev, Essentials of Fuzzy Modeling and Control. New York: Wiley, 1994.

[3] E. H. Mamdani and S. Assilian, "An experiment in linguistic synthesis with a fuzzy logic controller,” Int. J. Man-Mach. Stud., vol. 7, pp. 1-13, 1975.

[4] R. Babuska, Fuzzy Modeling for Control. Boston, MA: Kluwer, 1998.

[5] N. Kasabov, Foundations of Neural Networks, Fuzzy Systems and Knowledge Engineering. Cambridge, MA: MIT Press, 1996.
[6] B. Fritzke, "Growing cell structures_-A self-organizing network for unsupervised and supervised learning," Neural Netw., vol. 7, no. 9, pp. 14411460, 1994.

[7] N. K. Kasabov and Q. Song, "DENFIS: Dynamic evolving neural-fuzzy inference system and its application for time-series prediction," IEEE Trans. Fuzzy Syst., vol. 10, no. 2, pp. 144-154, Apr. 2002.

[8] P. Angelov, Evolving Rule-based Models: A Tool for Design of Flexible Adaptive Systems. Heidelberg, Germany: Springer-Verlag, 2002.

[9] R. Yager, "Learning methods for intelligent evolving systems," in Key Note Talk, Proc. Int. Symp. Evol. Fuzzy Syst. (EFS 2006). Piscataway, NJ: IEEE Press, pp. 3-7.

[10] P. Angelov and D. Filev, "An approach to online identification of TakagiSugeno fuzzy models," IEEE Trans. Syst., Man, Cybern. B, Cybern., vol. 34, no. 1, pp. 484-498, Feb. 2004.

[11] E. Lima, F. Gomide, and R. Ballini, "Participatory evolving fuzzy modeling," in Proc. Int. Symp. Evol. Fuzzy Syst. (EFS 2006). Piscataway, NJ: IEEE Press, pp. 36-41.

[12] E. Lughofer, E. Hüllermeier, and E. P. Klement, "Improving the interpretability of data-driven evolving fuzzy systems," in Proc. Eur. Soc. Fuzzy Logic Technol. (EUSFLAT 2005), Barcelona, Spain, pp. 2833.

[13] J. C. Barros and A. L. Dexter, "An evolving fuzzy model for embedded applications," in Proc. Int. Symp. Evol. Fuzzy Syst. (EFS 2006). Piscataway, NJ: IEEE Press, pp. 49-54.

[14] A. Kordon, "Inferential sensors as potential application area of intelligent evolving systems," presented at the 2006 IEEE Int. Symp. Evol. Fuzzy Syst. (EFS 2006).

[15] P. Angelov, D. Filev, N. Kasabov, and O. Cordon, Eds., "Evolving fuzzy systems," in Proc. Int. Symp. Evol. Fuzzy Syst. (EFS 2006). Piscataway, NJ: IEEE Press, pp. 1-372.

[16] F. Hoffmann, O. Cordon, P. Angelov, and F. Klawonn, in Proc. 3rd Int. Workshop Genet. Evol. Fuzzy Syst., Piscataway, NJ: IEEE Press, Mar. pp. 1-107.

[17] W. Pedrycz, Knowledge-Based Clustering: From Data to Information Granules. $\quad$ Chichester, U.K.: Wiley-Intersciense, 2005, pp. 1-316.

[18] N. Kasabov, Evolving Connectionist Systems. New York: SpringerVerlag, 2007.

[19] P. Angelov and X.-W. Zhou, "Evolving fuzzy classifier for real-time novelty detection and landmark recognition by a mobile robot," in Mobile Robots: The Evolutionary Approach (Studies in Computational Intelligence Series), N. Nedja, L. Coelho, and L. Mourelle, Eds. New York: Springer-Verlag, Mar. 2007, pp. 95-124.

[20] J. J. Macias-Hernandez, P. Angelov, and X. Zhou, "Soft sensor for predicting crude oil distillation side streams using Takagi-Sugeno evolving fuzzy models," in Proc. 2007 IEEE Int. Conf. Syst., Man, Cybern., Montreal, Canada, pp. 3305-3310. 